PROCEEDINGS OF THE

AMERICAN MATHEMATICAL SOCIETY

Volume 125, Number 2, February 1997, Pages 541-546

S 0002-9939(97)03714-3

\title{
RADIAL LIMIT OF LACUNARY FOURIER SERIES WITH COEFFICIENTS IN NON-COMMUTATIVE SYMMETRIC SPACES
}

\author{
CAMIL MUSCALU
}

(Communicated by Palle E. T. Jorgensen)

\begin{abstract}
Let $E$ be a rearrangement invariant space, $\Lambda \subseteq \mathbb{Z}$ an arbitrary set and $(M, \tau)$ a von Neumann algebra with a semifinite normal faithful trace. It is proved that the associated symmetric space of measurable operators $E(M, \tau)$ has $\Lambda$-RNP if and only if $E$ has $\Lambda$-RNP extending in this way some previous results by $\mathrm{Q}$. $\mathrm{Xu}$.
\end{abstract}

\section{INTRODUCTION}

The aim of the present note is to solve a problem concerning the $\Lambda$-Radon Nikodym property (in short $\Lambda$-RNP) in symmetric spaces of measurable operators. For stating our result we shall introduce first the necessary notations and definitions. Let $\Lambda \subseteq \mathbb{Z}$ be an arbitrary set. A Banach space $B$ is said to have $\Lambda$-RNP if and only if every $B$-valued bounded lacunary Fourier series $f\left(r e^{i t}\right)=\sum_{n \in \Lambda} a_{n} r^{|n|} e^{i n t}$ on the unit disc $D$ in the complex plane has radial limit at the boundary almost everywhere. As was proved in the paper of Buchvalov and Danilevich $[\mathrm{BD}]$ we have $\mathbb{Z}-\mathrm{RNP}=\mathrm{RNP}$, the usual Radon Nikodym property and also $\mathbb{N}$-RNP $=\mathrm{ARNP}$, the so-called analytic Radon Nikodym property. We consider now $(M, \tau)$ a semifinite von Neumann algebra acting on a Hilbert space $H$, with a normal faithful trace $\tau$. Let $\bar{M}$ be the space of all measurable operators with respect to $(M, \tau)$ in the sense of [N] equipped with the measure topology defined there. For $a \in \bar{M}$ and $t>0$ the $t$-th singular number of $a$ is defined by (cf. [FK])

$$
\mu_{t}(a)=\inf \{\|a e\| ; e \text { is a projection in } M, \tau(1-e) \leq t\} .
$$

The function $t \rightarrow \mu_{t}(a)$ will be denoted by $\mu(a)$. For the main properties of this function the reader is referred to $[\mathrm{FK}]$. Let also $E$ be a rearrangement invariant (r.i.) function space on $(0, \infty)$ (cf. [LT]). We define the non-commutative symmetric space associated with $(M, \tau)$ and $E$ as follows (cf. [DDP1]):

$$
\begin{gathered}
E(M, \tau)=\{a \in \bar{M} ; \mu(a) \in E\}, \\
\|a\|_{E(M, \tau)}=\|a\|_{E}=\|\mu(a)\|_{E}, a \in E(M, \tau) .
\end{gathered}
$$

More exactly, we shall consider throughout this paper the following two cases in order to preserve the main situations from the commutative case. If $(M, \tau)$ is

Received by the editors September 5, 1995.

1991 Mathematics Subject Classification. Primary 47B10, 47B35.

Key words and phrases. Lacunary Fourier series, Measurable operators. 
diffuse (cf. [SZ]) then $E$ is taken to be a r.i. space on $[0, \tau(1))$ and when $(M, \tau)$ is completely atomic with all the minimal projections of the same trace, $E$ will be a r.i. sequence space (cf. [LT]).

The problem we are mainly concerned here is if $\Lambda$-RNP for $E$ is equivalent with $\Lambda$-RNP for $E(M, \tau)$. Actually, in this paper we settle this question in the affirmative. In the particular cases $\Lambda=\mathbb{Z}$ and $\Lambda=\mathbb{N}$ this study was done in [X2] and $[\mathrm{X} 1]$ respectively. In [X1] the author also considered some other cases such as uniform convexity (see also $[\mathrm{M}]$ ), uniform PL-convexity and uniform H-convexity. Note however that such a result does not hold for AUMD property which does not pass from $E$ to $E(M, \tau)$ even in the particular case of the Schatten class (cf. [HP]). Also there exists $E_{1}$ isomorphic with $E_{2}$ and such that $E_{1}(M, \tau)$ is not equal with $E_{2}(M, \tau)$ (cf. [A] where such an example is given for $M=B(H)$ ). The arguments from $[\mathrm{X} 1, \mathrm{X} 2]$ do not seem to work in this more general case. Our solution is based on some recent results of the author in $[\mathrm{M}]$ and also on a compactness theorem in [DDP2].

\section{THE PROOF}

Let $\mathbf{T}$ be the unit circle equipped with its normalised Haar measure $d m$. We recall first that a vector measure $F:(T, d m) \rightarrow B$ has $L^{\infty}$-bounded variation (cf. [DU]) if and only if there exists $C>0$ such that $\|F(A)\|_{B} \leq C \cdot m(A)$ for every measurable subset $A$ of $\mathbf{T}$. The smallest constant for which the inequality holds is called the $L^{\infty}$-norm of $F$. For $\Lambda \subseteq \mathbb{Z}$ we shall denote by $V_{\perp}^{\infty}(B)$ the space of all vector measures $F$ with $L^{\infty}$-bounded variation and for which the Fourier coefficients

$$
\widehat{F}(n)=\int e^{-i n t} d m(t)=0
$$

for all $n \in \mathbb{Z} \backslash \Lambda$. $F$ is said to be representable if there exists $f \in L^{\infty}(B)$ such that $F=f \cdot d m$. Also, we define $H_{\Lambda}^{\infty}(B)$ to be the Hardy space of all functions $f: D \rightarrow B$ of the form $f\left(r e^{i t}\right)=\sum_{n \in \Lambda} a_{n} r^{|n|} e^{i n t}$ endowed with the $L^{\infty}$-norm. For proving our main result we need the next lemma which can be proved as in [Bl].

Lemma 2.1. Let $\Lambda \subseteq \mathbb{Z}$ and $B$ be an arbitrary Banach space. Then,

(a) $\quad H_{\Lambda}^{\infty}(B)=V_{\Lambda}^{\infty}(B)$ via the Poisson integral.

(b) $B$ has $\Lambda-R N P$ iff every $F \in V_{\Lambda}^{\infty}(B)$ is representable.

We can present now the main theorem in this paper as follows.

Theorem 2.2. We consider $\Lambda \subseteq \mathbb{Z}$ as above. A rearrangement invariant space $E$ has $\Lambda-R N P$ if and only if its non-commutative analogue $E(M, \tau)$ has $\Lambda-R N P$.

Proof. We shall give the proof in the "continuous case" when $(M, \tau)$ is diffuse. The "discrete case" can be done in the same way with some minor natural changes. Since $E$ is isometric isomorphic with a closed subspace of $E(M, \tau)$ it is sufficient to prove just " $E$ has $\Lambda$-RNP $\Rightarrow E(M, \tau)$ has $\Lambda$-RNP". We can assume card $\Lambda=\infty$ (if not, all Banach spaces have $\Lambda$-RNP) and remark as in [BD] that $E$ does not contain any copy of $c_{0}$. In particular the dual space $E^{*}$ is equal with the associate space $E^{\prime}$ (cf. [LT]). We consider first the situation $\tau(1)<\infty$ and we take $F \in V_{\Lambda}^{\infty}(E(M, \tau))$. To settle our problem we have to show that $F$ is representable (cf. lemma 2.1) or more particular, $R e F$ is representable $(F=R e F+i \operatorname{Im} F)$. Using a result from [DU] 
it is also sufficient to prove that for any $A \subseteq T, m(A) \neq 0$, there exists $A_{0} \subseteq A$, $m\left(A_{0}\right) \neq 0$ such that the set

$$
\mathcal{A}\left(A, A_{0}\right)=\left\{\frac{\operatorname{Re} F(B)}{m(B)} ; B \subseteq A_{0}, m(B) \neq 0\right\}
$$

is relatively weakly compact or, equivalently, relatively $\sigma\left(E(M, \tau), E^{\prime}(M, \tau)\right)$ compact by a result from [DDP2]. Anyway, since $(T, d m)$ is countable generated we deduce (cf. [DU]) that the range of $R e F$ is included into a separable subspace of $E(M, \tau)$. By a density result in $[\mathrm{M}]$ this implies that there exists a countable set $\mathcal{C} \subseteq E(M, \tau)$ such that every $T \in \mathcal{C}$ is selfadjoint, has $\mu(T)$ invertible and every element in $\overline{\operatorname{Sp}(\operatorname{range}(\operatorname{ReF}))}$ can be approximated in the norm of $E(M, \tau)$ by a net of measurable operators from $\mathcal{C}$. Let's say $\mathcal{C}=\left(T_{n}\right)_{n}$. For every $n \in \mathbb{N}$ there exists (cf. $[\mathrm{M}]$ ) a bounded linear map

$$
\Phi_{n}: E(M, \tau) \rightarrow E
$$

with $\Phi_{n}\left(\left|T_{n}\right|\right)=\mu\left(T_{n}\right)$ and the norms of $\Phi_{n}$ do not depend on $n \in \mathbb{N}$. Also, we define as in $[\mathrm{M}]$

$$
\begin{gathered}
\Psi_{n}: E \rightarrow E(M, \tau), \\
\Psi_{n}(f)=f \circ \mu^{-1}\left(T_{n}\right)\left(\left|T_{n}\right|\right)
\end{gathered}
$$

and recall (cf. $[\mathrm{M}])$ that $\mu\left(\Psi_{n}(f)\right)=f^{*}$, the usual nonincreasing rearrangement of $|f|$ (cf. [LT]). This means in particular that the space $E_{n}=\Psi_{n}(E) \subseteq E(M, \tau)$ is in fact a "copy" of $E$ which "stays" on the "direction" of $\left|T_{n}\right|$. After this preparation, we can start the proof of (2.1). So, let's fix $A \subseteq T, m(A) \neq 0$ and we want to define $A_{0}$. For this, we also consider $n \in \mathbb{N}$ and since $E_{n}$ has $\Lambda$-RNP it follows (cf. lemma 2.1) that the measure $\Psi_{n} \circ \Phi_{n} \circ R e F$ is representable. We obtain by a result from [DU] that for every $\epsilon>0$ and every $C \subseteq T, m(C) \neq 0$ there exists $C_{0} \subseteq C$, $m\left(C_{0}\right) \neq 0, m\left(C \backslash C_{0}\right)<\epsilon$, such that

$$
\mathcal{A}^{n}\left(C, C_{0}\right)=\left\{\frac{\Psi_{n} \circ \Phi_{n} \circ \operatorname{ReF}(D)}{m(D)} ; D \subseteq C_{0}, m(D) \neq 0\right\}
$$

is relatively norm compact in $E_{n}$. Now, it is obvious to choose a set $A_{0} \subseteq A$, $m\left(A_{0}\right) \neq 0$, with the property that for every $n \in \mathbb{N}$ the set $\mathcal{A}^{n}\left(A, A_{0}\right)$ is relatively norm compact in $E_{n}$. We will show that this $A_{0}$ is a good set for our problem. To see that $\mathcal{A}\left(A, A_{0}\right)$ is relatively $\sigma\left(E(M, \tau), E^{\prime}(M, \tau)\right)$ compact is equivalent (using a result from [DDP2] since $\tau(1)<\infty)$ to proving the following assertion:

"For every $x \in E^{\prime}(M, \tau)$ and $\left(x_{n}\right)_{n} \subseteq \Omega(x):=\left\{z ; \int_{0}^{t} \mu_{s}(z) d s \leq \int_{0}^{t} \mu_{s}(x) d s\right\}$ with $x_{n} \rightarrow x(\mu)$ we have

$$
\sup \left\{\int_{0}^{\infty} \mu_{t}\left(x_{n}\right) \mu_{t}(y) d t ; y \in \mathcal{A}\left(A, A_{0}\right)\right\} \rightarrow 0 . "
$$

We assume that the above statement is false. This means that there exists $x \in$ $E^{\prime}(M, \tau),\left(x_{n}\right)_{n} \subseteq \Omega(x), x_{n} \rightarrow 0(\mu)$ and $\epsilon>0,\left(y_{n}\right)_{n} \subseteq \mathcal{A}\left(A, A_{0}\right)$ such that

$$
\int_{0}^{\infty} \mu_{t}\left(x_{n}\right) \mu_{t}\left(y_{n}\right) d t>2 \epsilon, \quad n=1,2, \ldots
$$


Let $y_{n}=\frac{\operatorname{Re} F\left(B_{n}\right)}{m\left(B_{n}\right)}, n \in \mathbb{N}$. Arguing as in $[\mathrm{M}]$ it is not difficult to see that there exists $\left(y_{n}^{1}\right)_{n} \subseteq E_{1}$ with $\tau\left(y_{n} a\right)=\tau\left(y_{n}^{1} a\right)$ for every $a \in E_{1}^{\prime}\left(=\Psi_{1}\left(E^{\prime}\right)\right), n \in \mathbb{N}$, and

$$
\mu\left(y_{n}^{1}\right)=\mu\left(\frac{\Psi_{1} \circ \Phi_{1} \circ \operatorname{Re} F\left(B_{n}\right)}{m\left(B_{n}\right)}\right), n \in \mathbb{N} .
$$

Using the same compactness result from [DDP2] (see the above "assertion") together with (2.2) we obtain a subnet $\left(y_{k_{n}^{1}}^{1}\right)_{n} \subseteq\left(y_{n}^{1}\right)_{n}$ which converges weakly on the "direction" of $\left|T_{1}\right|$ (i.e. $\left(\tau\left(y_{k_{n}^{1}}^{1} a\right)\right)_{n}$ converges for every $\left.a \in E_{1}^{\prime}\right)$. This implies that $\left(y_{k_{n}^{1}}\right)_{n}$ converges weakly on the "direction" of $\left|T_{1}\right|$. In the same way we find a subnet $\left(y_{k_{n}^{2}}\right)_{n} \subseteq\left(y_{k_{n}^{1}}\right)_{n}$ which converges weakly on the "directions" of $\left|T_{1}\right|$ and $\left|T_{2}\right|$ and if we take the diagonal subnet we get in fact a subnet of $\left(y_{n}\right)_{n}$ which converges weakly on each "direction" $\left|T_{1}\right|,\left|T_{2}\right|, \ldots$ So, we can assume without loss of generality that $\left(y_{n}\right)_{n}$ itself has this property.

By the density of $\mathcal{C}$ in the norm of $E(M, \tau)$ and $(2.3)$, we can select a net $\left(T_{k_{n}}\right)_{n} \subseteq \mathcal{C}$ with the properties

$$
\int_{0}^{\infty} \mu_{t}\left(x_{n}\right) \mu_{t}\left(T_{k_{n}}\right) d t>2 \epsilon, n=1,2, \ldots
$$

and $\left\|y_{n}-T_{k_{n}}\right\|_{E(M, \tau)} \rightarrow 0$. Let us put $V_{n}:=T_{k_{n}}, n \in \mathbb{N}$. Since $(M, \tau)$ is diffuse it follows from (2.4) and [FK] that there exists $z_{n} \in E_{k_{n}}^{\prime}\left(:=\Psi_{k_{n}}\left(E^{\prime}\right)\right)$ with $\mu\left(z_{n}\right)=$ $\mu\left(x_{n}\right)$ and

$$
\left|\tau\left(z_{n} V_{n}\right)\right|>2 \epsilon, n=1,2, \ldots
$$

In general, the map $f:(\Omega(x), \mu) \rightarrow \mathbb{C}, f(z)=\tau(z y)$ is continuous for a fixed $y \in E(M, \tau)$ (cf. [DDP2]). But we know that $z_{n} \rightarrow 0(\mu)$ and that's why we can assume (tacking a subnet if necessary) that

$$
\left|\tau\left(z_{n+1} V_{n}\right)\right|<\epsilon, n=1,2, \ldots
$$

Since $\left\|y_{n}-V_{n}\right\|_{E(M, \tau)} \rightarrow 0$ it follows that $\left(V_{n}\right)_{n}$ also converges on each "direction" of $\left|T_{1}\right|,\left|T_{2}\right|, \ldots$. This means that if we let $w_{n}=V_{n}-V_{n-1}, n \in \mathbb{N}$, we have $\tau\left(w_{n} a\right) \rightarrow 0$ for every $a \in \bigcup E_{n}^{\prime}$. Using (2.5) and (2.6) we obtain

$$
\mid \tau\left(z_{n} w_{n}\right)>\epsilon, n=1,2, \ldots
$$

Let $Z=\left\{z_{n} ; n \in \mathbb{N}\right\} \bigcup\{0\}$. The space $(\Omega(x), \mu)$ is a complete metric space (cf. [DDP2] $)$ and so, $(Z, \mu)$ is a complete metric subspace of $(\Omega(x), \mu)$. So, if we define now the functions

$$
\begin{gathered}
f_{n}:(Z, \mu) \rightarrow \mathbb{C}, \\
f_{n}(z)=\tau\left(z w_{n}\right), z \in Z, n=1,2, \ldots,
\end{gathered}
$$

we deduce that $\left(f_{n}\right)_{n}$ are continuous functions and $f_{n}(z) \rightarrow 0$ for every $z \in Z$. Using Baire's theorem we obtain $N \in \mathcal{V}(0)$ in $(Z, \mu)$ and $n_{0} \in \mathbb{N}$ with the property $\left|\tau\left(z w_{n}\right)\right| \leq \frac{\epsilon}{2}$ for every $z \in N$ and $n \geq n_{0}$. But this implies that there exists $m_{0} \in \mathbb{N}$ such that

$$
\left|\tau\left(z_{m} w_{n}\right)\right| \leq \frac{\epsilon}{2}, \quad m \geq m_{0}, n \geq N_{0}
$$

which contradicts (2.7). The case $\tau(1)<\infty$ is proved. The general situation when $\tau(1)=\infty$ can be "reduced" to the first one by standard arguments. We give a sketch of proof. Let again $F \in V_{\Lambda}^{\infty}(E(M, \tau)$ ). First, using lemma 2.1 (a) we remark (cf. 
for instance [X2]) that we can assume without loss of generality that there exists a countable set of mutual disjoint projections $\left(P_{n}\right)_{n} \subseteq M, \tau\left(P_{n}\right)<\infty, n \in \mathbb{N}$, with $\sum_{n} P_{n}=1$. We put $Q_{n}=P_{1}+\ldots+P_{n}, n \in \mathbb{N}$. By the finite case, there exists $\phi_{n} \in L^{\infty}\left(E\left(Q_{n} M Q_{n}, \tau\right)\right), n \in \mathbb{N}$, with $Q_{n} F Q_{n}=\phi_{n} \cdot d m, n \in \mathbb{N}$. $E$ has a.c. norm (cf. [LT]); thus it is not difficult to see that $Q_{n} F(A) Q_{n} \rightarrow F(A)$ in $E(M, \tau)$ for any $A \subseteq T$ and also $\left(\phi_{n}(t)\right)_{n}$ is Cauchy in $E(M, \tau)$ a.e. $t \in T$. Let $\phi(t)=\lim _{n} \phi_{n}(t)$ a.e. $t \in T$. Since for every $A \subseteq T$ there exists $\lim _{n} \int_{A} \phi_{n}(t) d m(t)\left(=\lim _{n} Q_{n} F(A) Q_{n}=\right.$ $F(A)$ ) we deduce that $\phi$ is integrable (cf. [DU]) and moreover $F=\phi \cdot d m$ which completes the proof.

For $(M, \tau)=(B(H), t r)$ we get $E(M, \tau)=C_{E}$, the usual Schatten class, and we obtain the following consequences.

Corollary 2.3. We consider $\Lambda \subseteq \mathbb{Z}$. Then, $E$ has $\Lambda$-RNP if and only if $C_{E}$ has $\Lambda-R N P$.

Corollary 2.4 ([X1]). E has ARNP if and only if its non-commutative analogue $E(M, \tau)$ has ARNP.

Corollary 2.5 ([X2]). E has $R N P$ if and only if its non-commutative analogue $E(M, \tau)$ has $R N P$.

In $[\mathrm{BD}]$ it is proved that any Banach lattice not containing $c_{0}$ has ARNP. The following corollary can be considered as a non-commutative version of this result.

Corollary 2.6. A non-commutative symmetric space $E(M, \tau)$ has $A R N P$ if and only if it does not contain $c_{0}$.

Proof. The first "implication" is obvious since $c_{0}$ does not have ARNP. For the converse, let us observe that $E$ does not contain $c_{0}$ as well since it is isometric isomorphic with a subspace of $E(M, \tau)$. So, $E$ has ARNP (cf. [BD]) and then, by corollary 2.4 $E(M, \tau)$ has ARNP.

\section{REMARKS}

We want to point out here that the above results hold also in a more general context. For this, we have to recall the notion of Radon Nikodym Property associated with subsets of countable discrete abelian groups, introduced in [E] and [D]. Let $G$ be a compact abelian metrizable group, $\Gamma$ the dual group of $G, \mathcal{B}(G)$ the $\sigma$ algebra of Borel sets of $G$ and $\lambda$ the normalised Haar measure on $G$. We consider also $B$ a Banach space. If $\mu$ is a $B$-valued measure on $\mathcal{B}(G)$ and $\gamma \in \Gamma$ then the Fourier coefficient $\tilde{\mu}(\gamma)$ is defined by

$$
\tilde{\mu}(\gamma)=\int_{G} \overline{\gamma(x)} d \mu(x)
$$

If $\Lambda \subseteq \Gamma$ we denote as in [D] by $V_{\Lambda}^{\infty}(G, B)$ (resp. $\left.V_{\Lambda, \text { ac }}^{1}(G, B)\right)$ the set of vector measure with bounded $L^{\infty}$-variation such that $\tilde{\mu}(\gamma)=0$ for all $\gamma \in \Gamma \backslash \Lambda$ (resp. the set of vector measure with bounded variation, a.c. with $\lambda$ and having the same property). Following [E] and [D] we recall that $B$ is said to have I- $\Lambda$-RNP (resp. II$\Lambda$-RNP) if and only if every measure from $V_{\Lambda}^{\infty}(G, B)\left(\right.$ resp. $\left.V_{\Lambda, \text { ac }}^{1}\right)$ is representable. With the same arguments as above, the following theorem can be proven.

Theorem 3.1. With the above notations, a rearrangement invariant space $E$ has $I-\Lambda-R N P($ resp. II- $\Lambda-R N P)$ if and only if its non-commutative analogue $E(M, \tau)$ has I- $\Lambda$-RNP (resp. II- $\Lambda-R N P$ ). 


\section{REFERENCES}

[A] J.Arazy., Some remarks on interpolation theorems and the boundedness of triangular projection in unitary matrix spaces, Int. Eq. and Op. Theory 1/4 (1978). MR 81k:47056a

[Bl] O.Blasco., Boundary values of functions in vector valued Hardy spaces and geometry of Banach spaces, J.Func.Anal. 78 (1988), 346-364. MR 89f:46080

[BD] A.V.Buchvalov, A.A.Danilevich., Boundary properties of analytic and harmonic functions with values in Banach spaces, Mat.Zametki 31 (1982). MR 84f:46032

[DU] J.Diestel, J.J.Uhl., Vector measure, Providence, (1977).

[DDP1] P.G.Doods, T.K.Doods, B. de Pagter., Non-commutative Banach function spaces, Math. Z 201 (1989), 583-597. MR 90j:46054

[DDP2] _ Weakly compact subsets of symmetric operator spaces, Tech. rep., Delft University of Technology, (1990).

[D] P.N.Dowling., Radon-Nikodym properties associated with subsets of countable discrete abelian groups, Trans.Amer.Math.Soc. 327 (1991), 879-890. MR 92a:46019

[E] G.A.Edgar., Banach spaces with the analytic Radon Nikodym property and compact abelian groups, 1992.

[FK] T.Fack, H. Kosaki., Generalised s-numbers of $\tau$-measurable operators, Pacific J.Math. 123 (1986), 269-300. MR 87h:46122

[HP] U.Haagerup, G.Pisier., Factorization of analytic functions with values in noncommutative $L_{1}$-spaces and applications, Canad.J.Math 41 (1989), 882-906. MR 91g:46068

[LT] J.Lindenstrauss, L.Tzafiri., Classical Banach spaces, Springer-Verlag, 2 (1979). MR 81c: 46001

$[\mathrm{M}] \quad$ C.Muscalu., New results on interpolation in non-commutative analysis., preprint, (1995).

[N] E.Nelson., Notes on non-commutative integration., J.Func. Anal. 15 (1974), 103-116. MR 50:8102

[SZ] S.Stratila, L.Zsido., Lectures on Von Neumann algebras., Abacus Press (1979). MR 81j:46089

[X1] Q.Xu., Analytic functions with values in lattices and symmetric spaces of measurable operators., Math.Proc.Camb.Phil.Soc. 109 (1991), 541-563. MR 92g:46036

[X2] Q.Xu., Radon Nikodym property in symmetric spaces of measurable operators, Proc. Amer. Math. Soc 115 (1992), 329-335. MR 92i:46074

Institute of Mathematics of the Romanian Academy, RO70700, PO Box 1-764, BuchAREST, ROMANIA

E-mail address: muscalu@stoilow.imar.ro

Current address: Department of Mathematics, Brown University, Providence, Rhode Island 02912

E-mail address: camil@gauss.math.brown.edu 